http://dx.doi.org/10.21611/qirt.1994.010

\title{
Influence of changes of object-system distance on accuracy of remote temperature measurement
}

\author{
by CHRZANOWSKI K. and JANKIEWICZZ.
}

\author{
Military University of Technology, Institute of Optoelectronics, 01-489 Warsaw, Poland
}

\begin{abstract}
A study has been made to determine the influence of the difference between the distance object-system under calibration and the one at real work conditions on the accuracy of the temperature measurement with infrared systems. A theory of the influence of this difference on the errors of the temperature measurement for any measurement condition and system parameter has been developed. The results of the following calculation have shown that the errors of the remote temperature measurement due to this source can be significant
\end{abstract}

\section{Introduction}

It is a popular belief, created years ago [1], shared by many users of infrared systems and still sometimes reported in the literature [2] that, when the object is larger than the detector field of view and the influence of the atmosphere is negligible, the radiometric signal incident onto the detector does not depend on the distance object-system. Other researches [3] have reported the fact that the signal, or the system sensitivity, depends on the distance due to changes of the work conditions of the system optics. It has been concluded [3] that the problem of the influence of the distance on the measurement results can be eliminated by either performing the calibration and the measurement at the same distance, or by performing both at distances greater than the system hyperfocal distance.

However, in practice the situation looks completely different. The infrared systems are typically calibrated at one fixed distance between the system and the object, but the distance at real work conditions varies from a few tenth of a meter to thousands meters. The changes of the distance influence the absolute value of the signal measured by the system and cause measurement errors although, in spite of these changes, the operator of the system can still preserve a clear picture by adjusting the imaging optics of the system. For the case of long distance between the system and the tested object, even significant changes of the value of the distance have little influence on the measured signal. However, when the distance is short, as typical under many industrial conditions, even slight changes of it can strongly affect the value of the measured signal, and significantly reduce the accuracy of the temperature measurement.

In this paper an attempt has been made to quantitatively determine the effect of the difference between the distance object-system under calibration and the one at real work conditions on the accuracy of the temperature measurement with infrared systems. A theory of the influence of this difference on the errors of the temperature measurement, for any measurement condition and system parameter, has been developed.

The theory can be generally summarized with three primary formulas. The first one describes the influence of the distance object-system on the signal at the output of the IR detector. The second one describes the apparent errors of the signal measurement due to the difference between the distance object-system under calibration and the one at real work conditions. The third one describes the relationship between the errors of the signal measurement and the errors of the temperature measurement, so to allow to determine the last ones. On the basis of the developed formulas, an analysis of the influence of the difference between the distance object-system under calibration and the one at real work 
http://dx.doi.org/10.21611/qirt.1994.010

conditions, on the accuracy of temperature measurement for a few simulated systems, has been made.

\section{General theory.}

In this section, the basic principles involved in determining the relationship between the signal measured at the output of the detector and the distance between the tested object and the system are briefly reviewed. For this purpose several assumptions for the system are made in order to clarify the problems. First, the case of systems calibrated under laboratory conditions using an external reference source is considered. Second, the influences of the radiation reflected by the object and of transmittance and emittance of the atmosphere are assumed to be negligible. Third, it has been assumed that the optics and filters of the system can be treated as a single block of transmittance, this latter being equal to the product of the transmittances of each components. Fourth, the IR detector is put into the image plane of the tested object, and sees, due to the cold shield, only the optics.

The irradiation in the detector plane, for well-corrected imaging aplanatic optics that fulfills the sinus condition, can be expressed by the following formula [4]:

$$
E^{\prime}(\lambda)=\pi \tau_{o}(\lambda) \tau_{F}(\lambda) \varepsilon L_{e}(\lambda) \sin ^{2} u_{m}^{\prime},
$$

where $\tau_{0}(\lambda)$ is the optics transmittance at the wavelength $\lambda, \tau F(\lambda)$ the filter transmittance, $L_{\theta}\left(T_{o b}, \lambda\right)$ the object radiance at object temperature $T_{O b}$ and wavelength $\lambda, \varepsilon$ the object emissivity and $\sin u_{m}^{\prime}$ the numerical aperture of the optics in the imaging space.

The signal at the output of the detector is equal to

$$
S\left(T_{o b}^{\prime}\right)=\pi R_{\max } A_{d} c_{1} \varepsilon \sin ^{2} u_{m}^{\prime} \int_{\lambda_{1}}^{h_{1}^{2}} \frac{\tau_{o}(\lambda) \tau_{F}(\lambda) s(\lambda)}{\lambda^{5}\left[\exp \left(c_{2} / \lambda T_{o b}\right)-1\right]} d \lambda,
$$

where $A_{d}$ is the detector area, $R_{\max }$ the maximal detector sensitivity, $s(\lambda)$ the detector relative sensitivity, $\lambda_{1}$ and $\lambda_{2}$ the limits of the detector spectral band, $c_{1}$ and $c_{2}$ the Planck constants.

Eq.(1) has been developed for systems in the visible range while we must analyze the performance of systems in the infrared range. Eq.(1) has been determined on the basis of the optical rules that, in general, are also valid for the infrared systems. We can assume that, for systems in the visible, (or even near-infrared) range, only the radiation emitted by the object comes to the detector. However, we cannot do the same for systems of spectral bands located at longer wavelengths because we have to take into account the radiation emitted by the optics and filters that also comes to the detector $[5,6]$. The total amount of this radiation strongly depends on the detector field of view usually determined by the detector cold shield. Let us assume the case of ideal cold shielding, where the cold shield acceptance angle equals the angle subtended by the exit cone of the optics for a single, small detector. For the typical case of circularly symmetric optical system, the effective weighted solid angle is [7]

$$
\Omega_{D}=\pi\left(n \sin \left(\theta_{D} / 2\right)\right)^{2}=\frac{\pi}{4 F^{2}} \text { when } F \gg 0.5,
$$

where $\sin \left(\theta_{D} / 2\right)$ is the sine of the angle between the outermost (marginal) ray and the optical axis for the long distance object, and $F$ is the F-number of the imaging optics. By using the transfer equation [8] and the typical procedure, as employed earlier, for the radiation emitted by the object, the signal at the output of the detector due to the radiation emitted by the optics, can be determined as 
http://dx.doi.org/10.21611/qirt.1994.010

$$
S_{\text {opt }}=\frac{\pi R_{\max } A_{d} c_{1} \int_{\lambda_{1} \lambda^{5}\left[\exp \left(c_{2} / \lambda T_{o p t}\right)-1\right]}^{\left(1-\tau_{o}(\lambda) \tau_{F}(\lambda)\right) s(\lambda)} d \lambda}{4 F^{2}},
$$

where $T_{\text {opt }}$ is the optics temperature.

The total signal at the output of the detector is equal to the sum of the signal due to the radiation emitted by the object and the signal due to the radiation emitted by the optics and filters

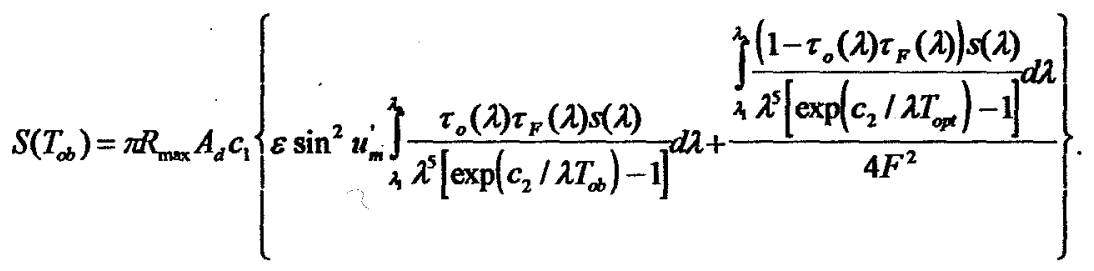

On the basis of geometry rules (Figure 1) and Newton law, we can write

$$
\sin u_{m}^{\prime}=\frac{\frac{D}{2}}{\sqrt{\left(f^{\prime}+\frac{f^{\prime 2}}{s-f^{\prime}}\right)^{2}+\left(\frac{D}{2}\right)^{2}}}
$$

where $f$ is the focal length of the imaging optics, $D$ is the diameter of the optics and $s$ is the distance between the optics and the tested object.

By using formula (6), we can transform formula (5) to a new form, as

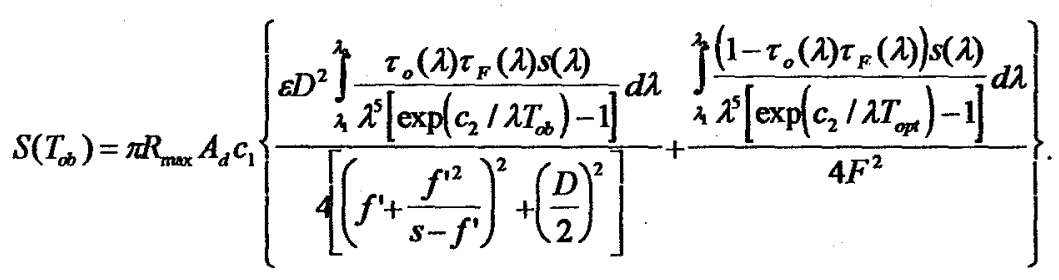

\section{Errors of signal measurement.}

It is a common practice that the users of the measuring infrared system calibrate these latter by using an external temperature reference source that simulates the tested object. The calibration process is typically carried out only for one fixed distance between the reference source and the system $s_{L}$, although the distance under real work conditions $s_{R}$ can vary from a few tenth of a meter to thousands meters. As Eq.(7) shows, the signal at the output of the detector $S$ depends on the distance object-system $S_{R}$. Therefore, the difference between the distance object-system under calibration and the one at real work conditions causes an apparent error of the signal measurement, that can be written as 
http://dx.doi.org/10.21611/qirt.1994.010

$$
\delta S=\frac{S\left(s_{R}\right)-S\left(s_{L}\right)}{S\left(s_{L}\right)},
$$

where the $\delta S$ is the relative error of the signal measurement. Putting the formula (7) into (8) we obtain

$$
\begin{aligned}
& \varepsilon D^{2} \int_{\lambda_{1}}^{\lambda_{1}} \frac{\tau_{o}(\lambda) \tau_{F}(\lambda) s(\lambda)}{\lambda^{5}\left[\exp \left(c_{2} / \lambda T_{o b}\right)-1\right]} d \lambda\left[\frac{1}{\left(f^{\prime}+\frac{f^{\prime 2}}{s_{R}-f^{\prime}}\right)^{2}+\left(\frac{D}{2}\right)^{2}}-\frac{1}{\left(f^{\prime}+\frac{f^{\prime 2}}{s_{L}-f^{\prime}}\right)^{2}+\left(\frac{D}{2}\right)^{2}}\right] \\
& \delta S= \\
& \frac{\varepsilon D^{2} \int_{\lambda_{1}}^{\lambda_{1}} \frac{\tau_{o}(\lambda) \tau_{F}(\lambda) s(\lambda)}{\lambda^{5}\left[\exp \left(c_{2} / \lambda T_{o b}\right)-1\right]} d \lambda}{\left(f^{\prime}+\frac{f^{\prime 2}}{s_{L}-f^{\prime}}\right)^{2}+\left(\frac{D}{2}\right)^{2}}+\frac{\int_{\lambda_{1}}^{\lambda^{5}\left[\exp \left(c_{2} / \lambda T_{o p t}\right)-1\right]}}{F^{2}}
\end{aligned}
$$

Let us now carry out a comparison of the performance of the three popular groups of systems measuring bands located in the infrared range: non-selective systems using thermal, or pyroelectric, detectors with filter of spectral band $8-12 \mu \mathrm{m}$; selective systems using photon detectors optimized for the 3-5 $\mu \mathrm{m}$ atmospheric windows ; systems of the $0.8-1.8 \mu \mathrm{m}$ spectral range designed using selective germanium Ge detector. As can be seen in figure 2, the influence of the difference between the distance object-system under calibration $s_{L}$ and the one at real work conditions $s_{R}$ on the relative errors of the signal measurement $\delta S$ significantly differs in the three compared systems. The influence is the strongest for the Ge systems, the weakest for the non-selective systems. The influence also depends on the object temperature, particularly for the non-selective systems. For these systems we can even say that the errors of the signal measurement $\delta S$, due to not too large changes of the distance object-system, can

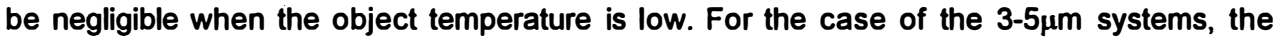
dependence of the errors $\delta S$ on the object temperature is significantly weaker, for Ge systems the dependence does not exist.

To summarize the situation we can say that, in general, the errors of the signal measurement $\delta S$, with the exception of the non-selective systems and low temperature object, are significant.

\section{Errors of temperature measurement.}

Formula (9) enables us to determine the apparent errors of the signal measurement $\delta S$ due to the difference between the distance object-system under calibration and the one at real work conditions. However, we are interested in the final effect, i.e. the errors of the temperature measurement $\delta T$. For the case of small changes in the measured signal $S$ and systems calibrated for long distance objects, the formula for the temperature measurement errors $\delta T$ can be written as [9]

$$
\delta T=\delta S \frac{\varepsilon \int_{\lambda_{1}}^{\lambda_{1}} \frac{\tau_{o}(\lambda) \tau_{F}(\lambda) s(\lambda)}{\lambda^{5}\left[\exp \left(c_{2} / \lambda T_{o b}\right)-1\right]} d \lambda+\int_{\lambda_{1}}^{\lambda} \frac{\left[1-\tau_{o}(\lambda) \tau_{F}(\lambda)\right] s(\lambda)}{\lambda^{5}\left[\exp \left(c_{2} / \lambda T_{o p t}\right)-1\right]} d \lambda}{T_{o b} c_{2} \varepsilon \int_{\lambda}^{\lambda_{1}} \frac{\tau_{o}(\lambda) \tau_{F}(\lambda) s(\lambda) \exp \left(c_{2} / \lambda T_{o b}\right)}{\lambda^{6} T_{o b}^{2}\left(\exp \left(c_{2} / \lambda T_{o b}\right)-1\right)^{2}} d \lambda}
$$


http://dx.doi.org/10.21611/qirt.1994.010

where $\delta T$ is the relative error of the temperature measurement.

Now, let us find out what are the errors of the temperature measurement $\delta T$ caused by the difference between the distance object-system under calibration and the one at real work conditions. The determined values of $\delta T$ caused by the errors of the signal measurement for the non-selective $(8-12 \mu \mathrm{m})$ and the selective $(3-5 \mu \mathrm{m})$ systems are presented in figure 3 . As we can see the difference between the errors of temperature measurement for the two compared systems is much smaller than the one for the errors of the signal measurement. This happens due to the influence of the function of the relationship between the errors of the signal and the temperature measurement that prefers short-wave systems [9]. We can even say, in general, that the situation appears the same for the two compared systems: the errors of the temperature measurement are small for low temperature objects, but are significant for high temperature ones.

\section{Conclusions.}

The results of this study have shown that the difference between the distance objectsystem under calibration and the one at real work conditions can lead to significant errors of the temperature measurement and the users should be aware of them.

For systems of the middle infrared range, the errors rise with the temperature of the tested object and, although small for low temperature objects, they are always significant for high temperature ones. Therefore the belief that, when the object is larger than the detector field of view, the radiometric signal incident onto the detector, and next the temperature indication, do not depend on the distance object-system can be justified only for the case of low temparature object and small changes of the distance object-system.

The developed formulas have been used to determine the errors of the signal and of the temperature measurement due to the difference between the distance object-system under calibration and the one at real work conditions. They can be used to correct these errors.

The conclusions presented herein have significant practical relevancy. However, one has to remember that they are limited to systems and measurement conditions that fulfill the assumptions which have been made. Further study is needed to explore the sensitivity of the conclusions when the above assumptions are not fulfilled.

\section{REFERENCES}

[1] GRIFFITHS (E.), Methods of measuring temperature, Chapt.5, 109, Charles Griffin \& Co., London (1947).

[2] FEHRIBAH (J.D.), JOHNSON (R.B.), Opt. Eng. 28, 1255 (1989).

[3] FRAEDRICH (S.), Opt. Eng. 30, 1764 (1988).

[4] JÓŸWICKI (R.), instrumental optics, Chapt. 2, PWN, Warsaw (1970).

[5] Wolfe (W.L.), In Applied Optics and Optical Engineering. Vol.8, Chapt. 5, Academic Press, New York (1980).

[6] WILLIAMS (O. M.), Infrared Phys. 26,141 (1986).

[7] BARHYDT (H.), Opt. Eng. 17, 28 (1998).

[8] ZISSIS (G.J.), In The Infrared \& Electro-Optical Systems Handbook, Vol.1:Sources of Radiation, Chapt. 1, 27-28, SPIE (1993).

[9] CHRZANOWSKI (K.), "Comparison of shortwave and longwave measuring thermal imaging systems, App. Opt. (accepted for publication) 


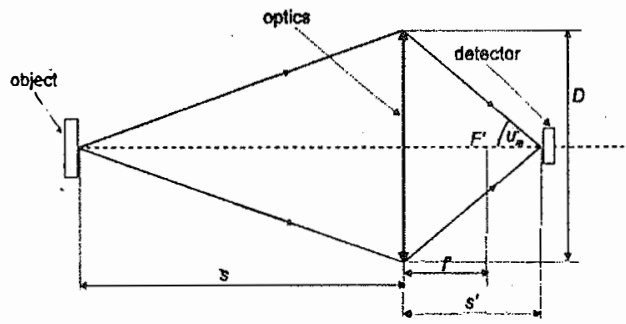

Fig.1. The scheme of creating the image of the tested object by optical system.

a)

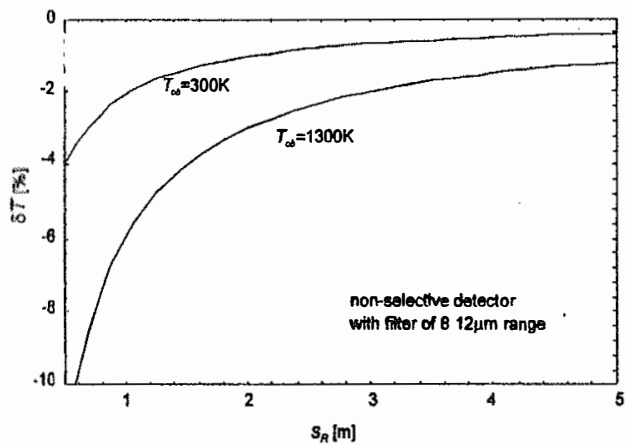

b)

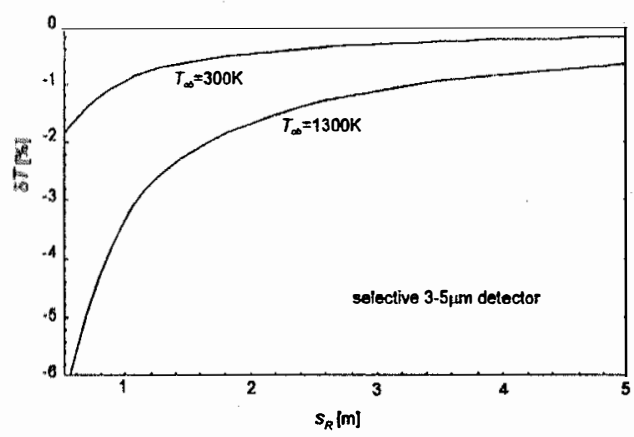

Fig. 3. Errors of temperature measurement $\delta T$ : a) non-selective detector with the filter of 8-12 $\mu \mathrm{m}$ spectral range, b) selective 3-5,1m detector. a)

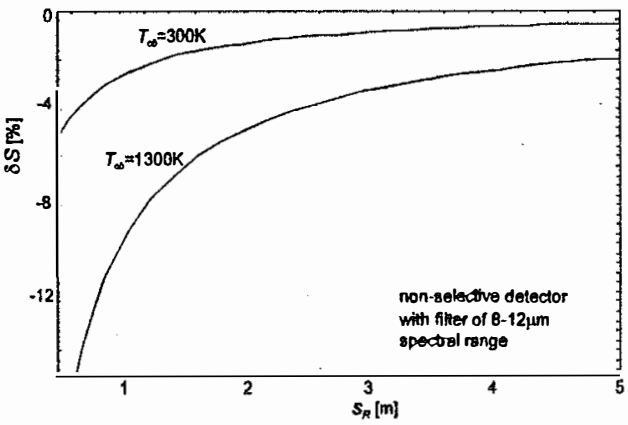

b)

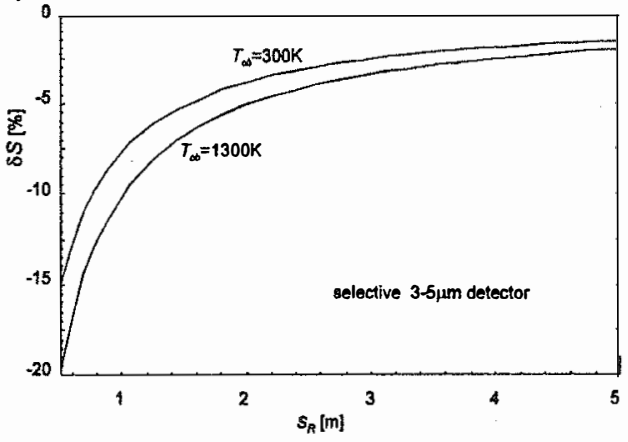

c)

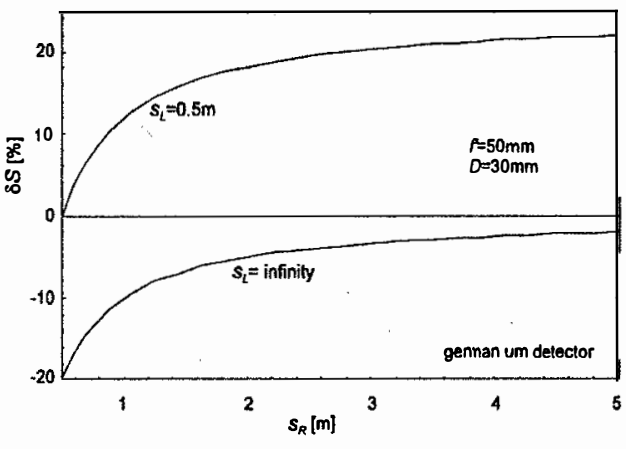

Fig.2. Errors of signal measurement $\delta S:$ a) non-selective detector with the filter of $8-12 \mu \mathrm{m}$ spectral range, b) selective detector optimized for the 3-5,ım "atmospheric window", c) germanium detector. 